\title{
Exploring the role of conceptual scaffolding in solving synthesis problems
}

\author{
Lin Ding, ${ }^{1, *}$ Neville Reay, ${ }^{2}$ Albert Lee, ${ }^{3}$ and Lei $\mathrm{Bao}^{2}$ \\ ${ }^{1}$ School of Teaching and Learning, The Ohio State University, Columbus, Ohio 43210, USA \\ ${ }^{2}$ Department of Physics, The Ohio State University, Columbus, Ohio 43210, USA \\ ${ }^{3}$ Department of Physics and Astronomy, California State University Los Angeles, Los Angeles, California 90032, USA
}

(Received 22 January 2011; published 14 October 2011)

\begin{abstract}
It is well documented that when solving problems experts first search for underlying concepts while students tend to look for equations and previously worked examples. The overwhelming majority of endof-chapter (EOC) problems in most introductory physics textbooks contain only material and examples discussed in a single chapter, rarely requiring a solver to conduct a general search for underlying concepts. Hypothesizing that complete reliance on EOC problems trains students to rely on a nonexpert approach, we designed and implemented "synthesis" problems, each combining two major concepts that are broadly separated in the teaching timeline. To provide students with guided conceptual scaffolding, we encapsulated each synthesis problem into a sequence with two preceding conceptually based multiplechoice questions. Each question contained one of the major concepts covered in the subsequent synthesis problem. Results from a small-scale interview study and two large-scale written tests showed that the scaffolding encouraged students to search for and apply appropriate fundamental principles in solving synthesis problems, and that repeated training using scaffolded synthesis problems also helped students to make cross-topic transfers.
\end{abstract}

DOI: 10.1103/PhysRevSTPER.7.020109

PACS numbers: 01.40.Fk, 01.40.Ha

\section{INTRODUCTION}

An important goal of introductory physics is to train students to be competent problem solvers [1-6]. One feature characteristic of skilled problem solving is successive refinement that begins with a search for fundamental concepts [7-10]. Studies have shown that when solving a problem experts often qualitatively search for underlying concepts, followed by further elaboration at a more detailed level before performing quantitative analysis using mathematical equations [11-16]. In contrast, students frequently begin by searching for formulas and worked-out examples without recognizing deep structure (relevant concepts and principles) [11,12]. Among various types of problem-solving practices, traditional end-of-chapter exercises (EOCs) perhaps are most commonly used in introductory physics courses to train students in learning content. However, these EOCs often are localized, addressing only material covered in a single chapter $[2,17]$. When solving these problems, students are not motivated to search for underlying concepts, but rather are encouraged to look locally for formulas and worked-out examples and then do plug-and-chug to get a correct answer. As a result,

\footnotetext{
*Corresponding author. ding.65@ osu.edu.
}

Published by the American Physical Society under the terms of the Creative Commons Attribution 3.0 License. Further distribution of this work must maintain attribution to the author(s) and the published article's title, journal citation, and DOI. they frequently fail to develop an expertlike approach even after doing a large number of EOCs $[18,19]$.

Researchers have explored various ways to encourage students to rely on underlying concepts to solve physics problems. For example, Larkin [8] used "a programmed booklet, individual testing and remedial instruction" to train students to concentrate on physical principles in solving dc-circuit problems. Reif and Scott [20] designed a computer program entitled "personal assistance for learning" to promote important cognitive skills, including principle-oriented decision making. Dufresne et al. and Mestre and et al. [21,22] created a computer-based "hierarchical analysis tool" to facilitate student recognition of deep structure. Leonard et al. [23] implemented "strategy writing" to explicate underlying concepts involved in physics problems. Kanim employed "bridging exercises" to help students with concepts, thereby encouraging non-formula-driven approaches to electricity problems [24]. Most recently, a study by Singh [25] showed that when answering a qualitative question paired with a preceding isomorphic quantitative question, students could discern their underlying connections, a phenomenon bearing on analogical problem-solving techniques [26]. All of these studies suggest that effective problem-solving skills may be cultivated through target training.

On the basis of these studies, we designed and implemented problems containing multiple concepts that are broadly separated in the teaching timeline to foster effective problem-solving skills among introductory students. These problems, which we name "synthesis problems," cannot be easily solved by simply invoking locally 
A small object attached to a spring of stiffness $k$ is oscillating on a smooth surface, where the frictional force between the object and the surface is negligible. Initially the mechanical energy of the spring-object system is $E_{i}$. After the block travels a distance of $d$, how does the total mechanical energy of the spring-object system change?

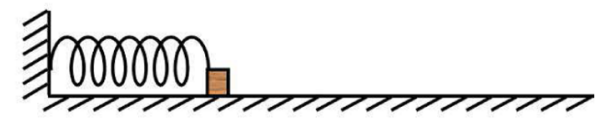

(1) It remains the same: $E_{i}$

(2) It increases by a value of $k d^{2}$

(3) It increases, but the increased value is unknown

(4) It decreases by a value of $k d^{2}$

(5) It decreases, but the decreased value is unknown

(a)

A small object attached to a spring is oscillating on a rough surface, where the frictional force between the object and the surface is a constant $f$. Initially the mechanical energy of the spring-object system is $E_{i}$. After the block travels a distance of $d$, how does the total mechanical energy of the spring-object system change?

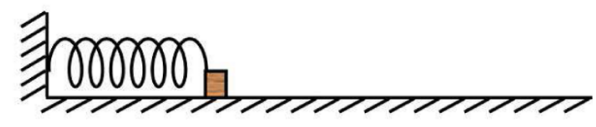

(1) It remains the same: $E_{i}$

(2) It increases by a value of $f d$

(3) It increases, but the increased value is unknown

(4) It decreases by a value of $f d$

(5) It decreases, but the decreased value is unknown

(b)

A small ball of mass $0.05 \mathrm{~kg}$ is attached to a spring of stiffness $2 \mathrm{~N} / \mathrm{m}$, and it oscillates along the $x$ axis on a rough surface. Initially the maximum spring stretch is 1 $m$. Due to the friction between the ball and the table surface, the maximum stretch of the spring gets smaller. After traveling a distance of $3.8 \mathrm{~m}$, the ball is at the position $x=+0.6 \mathrm{~m}$. At this moment what is the speed of the ball? The frictional coefficient between the ball and the table surface is $\mu_{\text {kinetic }}=\mu_{\text {static }}=\mathbf{0 . 2}$.

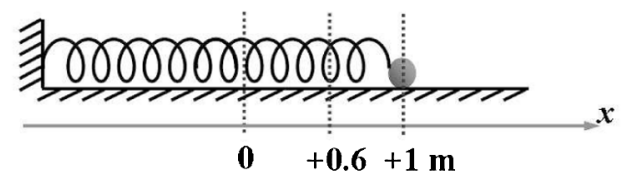

(c)

FIG. 1 (color online). A sequence example: (a) and (b) are two conceptually based multiple-choice questions; (c) is an openended problem that synthesizes broadly separated topics in teaching materials. We deliberately used the phrase of "mechanical energy" in (a) and (b) to distinguish it from "energy" of a system, as the former is conserved only in the absence of dissipative forces while the latter is always conserved regardless. introduced formulas, and hence they can be used to militate against simple "plug-and-chug." To further induce students to search for relevant conceptual knowledge before using mathematical formulas, we encapsulated each synthesis problem into a sequence with two preceding conceptually based multiple-choice questions. These concept questions share with the subsequent problem the same deep structure and serve as guided scaffolding to stimulate students' consideration of fundamental concepts. A sample sequence is shown in Fig. 1. The synthesis problem in Fig. 1(c) involves both the work-energy theorem and friction in oscillatory motion-two concepts introduced, respectively, in the 1st and 3rd quarters of a calculus-based introductory physics course taught at The Ohio State University (OSU). Before attempting to solve the synthesis problem, students are advised to seek its connections with the concept questions [Figs. 1(a) and 1(b)]. However, students must determine the connections by themselves.

\section{RESEARCH QUESTIONS}

As a primary endeavor, we implemented several sequences in a small-scale interview study and two large-scale written tests to explore both the immediate and sustained effects of the conceptual scaffolding on student solving synthesis problems. This paper reports results from these studies. Specifically, we attempted to answer the following three research questions. (1) How do students receiving guided conceptual scaffolding perform on the synthesis problem as opposed to those who do not receive the scaffolding? (2) What differences does it make to provide students with direct cueing instead of conceptual scaffolding? Here, direct cueing means explicitly telling students the relevant concepts required for solving a problem. (3) What effects does repeated training with scaffolded synthesis problems engender, especially after the scaffolding is removed?

The following sections (Secs. III, IV, and V) are devoted to the procedures and results of the three studies. Conclusions and discussion are presented in Sec. VI.

\section{STUDY 1: INVESTIGATING IMMEDIATE EFFECTS OF SCAFFOLDING IN SMALL-SCALE INTERVIEWS}

\section{A. Procedure}

Private one-on-one interviews were conducted in the spring quarter of 2008 using the sequence shown in Fig. 1. Twelve paid students who were recruited on a voluntary basis participated in the study. These students were from a then on-going 3rd quarter calculus-based introductory physics course. Before the interviews, students all had learned waves and oscillatory motions in class and practiced on relevant concepts in recitation and homework. We therefore were interested to see how students would be able to tackle a synthesis problem 
combining this topic with another one that was learned in the 1st quarter physics course: the work-energy theorem. We randomly divided the 12 recruited students into two groups using a blind design: "scaffolding group" (SG) and "plain problem group" (PPG), with 6 in each. Those in the SG group first answered the two conceptual questions in Figs. 1(a) and 1(b) and then solved the subsequent problem [Fig. 1(c)]. Before embarking on the synthesis problem, students were also told that they "may look for underlying connections between the two questions and the problem at hand." But they were neither confirmatively assured that there must exist a connection nor were they told what the connections, if any, would be. To reduce unnecessary "study availability effects" (due to the proximity in time between scaffolding and test problem) [27], we purposely used a suggestive rather than an imperative statement to point out that students had an option to look for underlying connections. Besides this reminder, no additional feedback of any form (on the scaffolding questions or on the synthesis problem) was provided. Conversely, the PPG students were asked to solve the synthesis problem without being given the preceding questions. Students in both groups were allowed to use their textbooks, notebooks, and calculators.

During the interviews, students were required to talk aloud, and the interviewer provided minimal interventions only for reminding students to keep talking or asking them to clarify explanations not understood by the interviewer. Enough time was given to each student for the required task. Each session was concluded either when a student successfully finished the task or decided to stop. All interview sessions were video recorded and later transcribed. The students' written work during the interviews was also collected for analysis.

\section{B. Results}

The two conceptual questions were shown to be fairly straightforward, and all SG students answered correctly with appropriate reasoning. For brevity and for the purpose of the study, we thereafter concentrate on student performance in solving the open-ended synthesis problem.

The interviews showed that all SG students were able to use the correct fundamental concepts (the work-energy theorem in connection with frictional force in oscillatory motion) to tackle the synthesis problem without referring to their textbooks or notes. Though some students made calculation errors and failed to obtain a correct final answer, all six recognized the connections of the preceding questions with the problem. The average time that the SG students took to identify these connections was $33 \mathrm{sec}$. In general, these students focused mainly on the fundamental concepts rather than on specific terms or their mathematical expressions. They correctly identified the initial and final states of the ball-spring system, enumerated corresponding energy forms, and attributed the decrease in the system's mechanical energy to the work done by the frictional force in oscillation. The following excerpt illustrates a typical comment made by the SG students: "We don't know whether or not this $(x=0.6 \mathrm{~m})$ is when the spring is at its maximum compression... But we can use the ideas from last two questions that mechanical energy may not be conserved, but we can figure out what the work is done by friction. And because of that, then we can figure out what change in kinetic energy is at any point." Based on these connections, all SG students then used a "forward" strategy [28] to solve the problem, working top-down from the known variables to the unknowns [29].

In contrast, none of the students in the PPG used the fundamental concepts as an initial approach and eventually failed to solve the problem. Although one student struggled to relate the problem to the work-energy theorem in the very late stage of her interview session, she was still unable to use it properly. During the debriefing after the interviews, we asked each PPG student to determine the speed of the ball at some intermediate position in the absence of friction; all could answer it correctly. But combining friction in oscillatory motion and the work-energy theorem proved difficult for them, and they resorted to novicelike strategies [30]. In particular, some students in the PPG immediately began to look into their textbooks or notes after reading the problem. Some specifically searched for formulas of damped oscillation, but soon came to a dead end [31]. They either could not make sense of the variables in the differential equation or could not solve it even if they knew what each variable represented. Some resorted to pattern matching, hoping to find similar worked-out examples in their textbooks [32]. But they quickly realized that this problem was not like the ones they saw in class.

TABLE I. Typical excerpts from the PPG students who approached the problem by equation search, pattern match, or random search.

\begin{tabular}{lcl}
\hline \hline $\begin{array}{l}\text { Type of search } \\
\text { sumber of } \\
\text { Equation search }\end{array}$ & 4 & Typical excerpts \\
$\begin{array}{l}\text { Pattern match } \\
\text { Random search }\end{array}$ & 2 & "I'm gonna just write down the equation for damped motion." \\
& 2 & "I'm gonna try to find a problem that's like this, an example like this..." \\
"If I had to start, I would probably just start charting down everything that was relevant
\end{tabular}

${ }^{\mathrm{a}}$ Some students used more than one type of search. 
Other students aimlessly flipped through their notes, hoping to find hints. (Table I shows typical excerpts for each type of search.) Since these students could not relate the given information with unknowns, they often relied on the "means-ends" $[28,33]$ approach to tackle the problem. Specifically, they started with an equation containing the speed variable and worked backwards to find more equations to solve for other unknowns in the equation. Then they worked forward to replace these unknowns in the preceding equations to finally find the speed of the ball. One interesting observation was that although the PPG students solved only one problem, the amount of time they spent was comparable to that by the SG students who solved the same problem plus two conceptual questions ( $\sim 20 \mathrm{~min})$. This was largely because the PPG students spent a considerable amount of time thumbing through textbooks and notes for ideas.

\section{STUDY 2: INVESTIGATING IMMEDIATE EFFECTS OF SCAFFOLDING IN A LARGE-SCALE WRITTEN TEST}

\section{A. Procedure}

A large-scale written test was conducted in the fall of 2008. The goal of this study was twofold. First, we wanted to see if results similar to study 1 would be obtained in a larger sample of students. Second, it was suggested that the effect of conceptual scaffolding used in the interviews might be equivalent to that of direct cueing [34]. If that were the case, then direct cueing alone would be sufficient to help students solve synthesis problems.

The questions and problem shown in Fig. 2 were used in a written test [35]. Here, the open-ended synthesis problem [Fig. 2(c)] addresses both linear and angular momentatwo topics that are taught, respectively, in the middle and at the end of the mechanics sequence at OSU [36]. In total, 360 students from 18 parallel recitation sections of a calculus-based introductory mechanics course took the written test and received credit for participation. We randomly divided these students into three groups by their recitation sections: SG, PPG, and "cueing group" (CG), with 143, 109, and 108 students in each. The SG students were first directed to answer the two conceptual questions in Figs. 2(a) and 2(b) either individually or collaboratively in $5 \mathrm{~min}$. Here, the quantitative nature of the first scaffolding question required students to use learned physics concepts rather than intuitive knowledge to answer it, whereas the second qualitative question avoided asking students to perform similar calculations needed for solving the subsequent synthesis problem. After answering the scaffolding questions, students were then asked to work independently on the synthesis problem within $12 \mathrm{~min}$. Similarly to study 1, the SG students were told that they "may look for underlying connections between the two questions and the problem." But they were not told whether or not such connections necessarily existed or what they would be.
On a frictionless surface, a car of mass $M$ is moving at a speed of $10 \mathrm{~m} / \mathrm{s}$ toward another stationary car of mass 2 M. Subsequently, they collide and stick together. What is the final speed of the two car system?

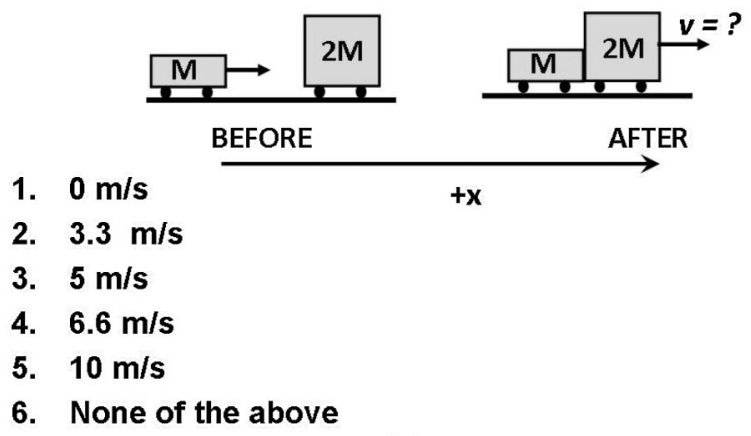

(a)

A merry-go-round is initially spinning clock-wise around its axial with negligible friction. A child is running at a certain speed towards the merry-go-round, jumps on and stays near its edge. Consider the child and the merry-go-round as a system. How does the angular momentum of the system change after the child jumps on the merry-go-round?

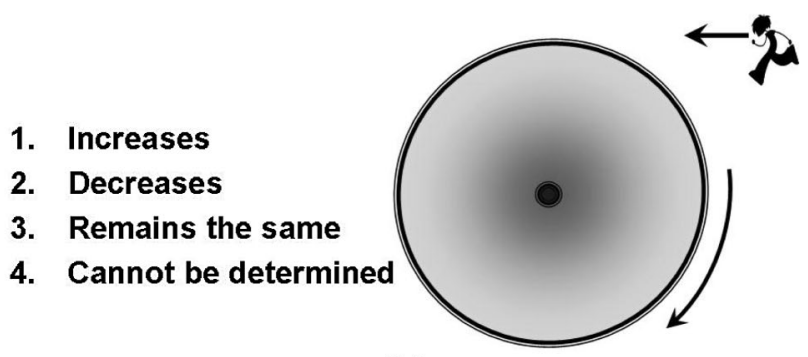

(b)

Tom throws a clay blob of mass $m$ at a massless "black clay catcher" located on the outer rim of a puck which in turn is riding on a horizontal frictionless air table. The clay is moving at a speed of $v$ in a direction tangent to the rim of the puck just before it sticks onto the catcher. Initially stationary, the system of puck plus clay after impact begins moving on the frictionless surface. Tom sees that the metal puck is a uniform disk with a mass of $M$ and a radius of $R$. Remember all uniform disks have a moment of inertia $1 / 2 M R^{2}$. What is the final total kinetic energy of the moving puck plus clay system?

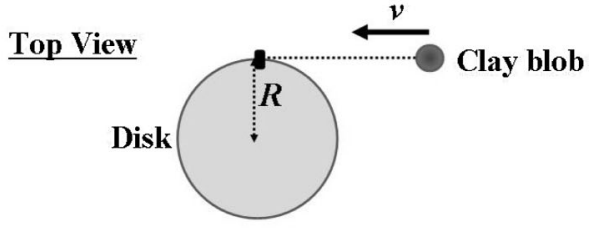

(c)

FIG. 2. A sequence used for a large-scale written test. (a) and (b) are conceptual questions; (c) is an open-ended synthesis problem. 
They received no additional feedback (on the scaffolding questions or on the synthesis problem) other than this reminder. Students in the PPG group were given $17 \mathrm{~min}$ to solve only the synthesis problem without answering the preceding questions. Similarly, the CG students also were allowed $17 \mathrm{~min}$ to solve the problem without answering the questions. But a direct cueing that "Momentum and angular momentum may play a role" was given in the problem statement. All groups were permitted to use their textbooks, notes, and calculators.

Since we were more interested in how students approached the problem than whether they obtained a final correct answer, we judged their written responses against two criteria: consideration and proper application of the conservation laws of linear and angular momenta. Specifically, we used the following rubrics to examine the presence or absence of the criteria.

(1) Consider fundamental concepts. - Did the students show written evidence of considering both linear and angular momenta, and did they state that linear and angular momenta are conserved? Responses of any forms, including words, symbols, or diagrams, that contained either general expressions of the conservation laws or specific equations with expanded terms would be considered as meeting this criterion.

(2) Meaningful expansion.-Did the students make a meaningful expansion of the fundamental concepts to determine the final linear and angular speed of the system? Given a proper "consideration of fundamental concepts," efforts to itemize and equate the system's initial linear or angular momentum to that of the final state would be acceptable. (For example, writing down the initial and final angular momenta and equating them to solve for the final angular speed.) No penalty was given for typographical and computational errors or translation of errors.

Two researchers independently examined student solutions, and agreed on $94 \%$ of the cases. The few disagreements were resolved with follow-up discussions.

\section{B. Results}

Student performance on the synthesis problem is depicted in Fig. 3, which shows the percentages of students in each group who considered and made meaningful expansion of the appropriate fundamental principles. As seen, more students in the SG and the CG started with the proper fundamental principles than those in the PPG. [SG: $t(250)=2.85, p=0.005$; CG: $t(215)=2.04, p=$ 0.042.] However, the percentages were comparable between SG and CG $[t(249)=0.67, p=0.50]$. This suggests that direct cueing and conceptual scaffolding were equally effective in prompting students to consider the relevant underlying principles. (A closer look at student responses further revealed that those who did not consider the conservation laws in the CG almost always dwelled on the definition of linear and angular momenta.) However,

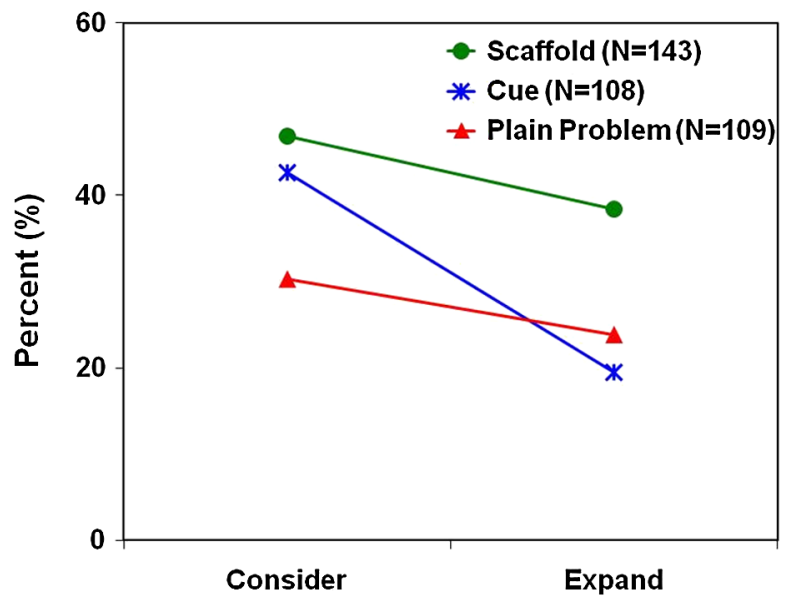

FIG. 3 (color online). Percentages of students who considered and made meaningful expansions of both of the conservation laws of linear and angular momenta.

Fig. 3 also shows that a larger fraction of students in the SG made a meaningful expansion of the fundamental concepts than in the CG and the PPG. [CG: $t(249)=3.19, p=$ 0.002; PPG: $t(250)=2.54, p=0.012$.] This indicates that although direct cueing is as helpful as scaffolding in getting students started with the underlying concepts, it is less effective in facilitating a sensible expansion of the cued concepts.

These results notwithstanding, it is possible that the initial assignment of students had been inadvertently biased in favor of the SG. In other words, higher-achieving students by chance had been assigned to the SG. To rule out this possibility, we collected and examined students' final course grades for the mechanics course they were then attending. As shown in Fig. 4, the average grade point was nearly the same across all groups $[F(2,351)=0.12$, $p=0.891]$.

To further examine how students of different grade levels performed on the synthesis problem, we divided students of each group into four achievement levels

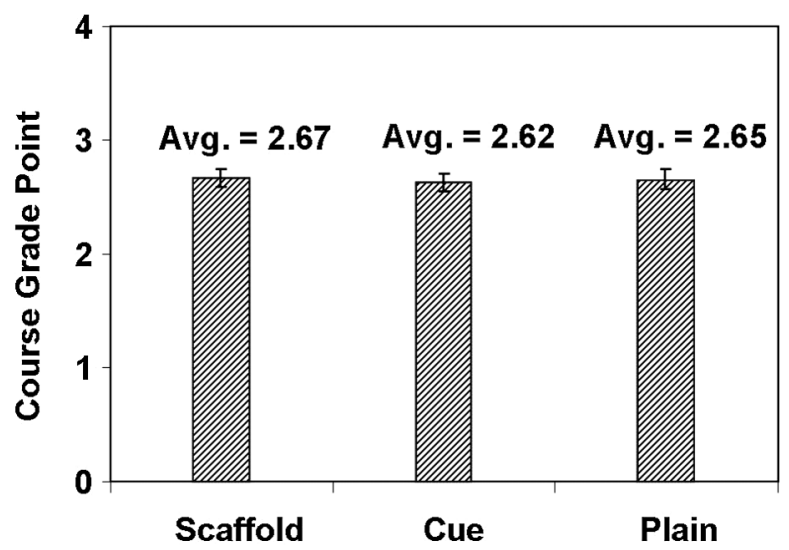

FIG. 4. Student grade points for a calculus-based introductory mechanics course. 


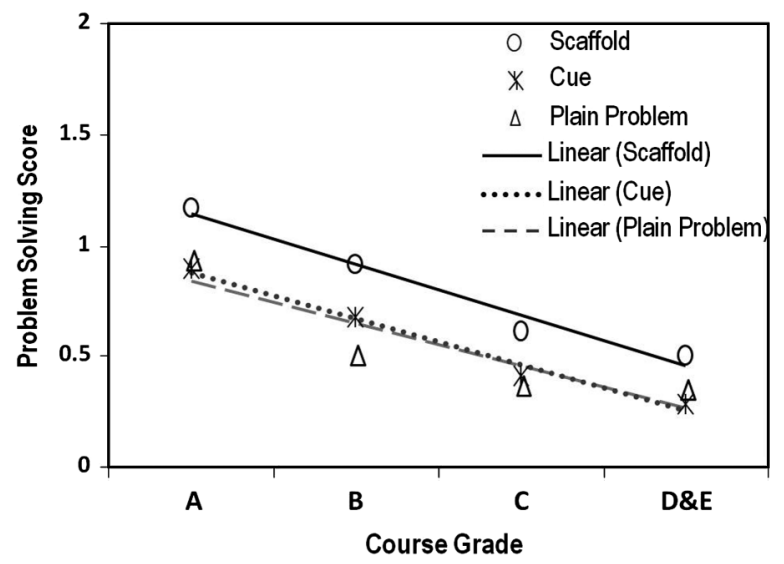

FIG. 5. Problem-solving performance for students at different course grade levels.

according to their final course grades: A, B, C, and D\&E [37]. Since students with a grade of either D or E accounted for less than $10 \%$ in each group, we combined them into one level. We then assigned a problem-solving score to each student using the aforementioned grading rubrics. Specifically, a score of 2 was given to those who both considered and made a meaningful expansion of the relevant fundamental concepts. Those who considered but failed to make a sensible expansion of the appropriate concepts received a score of 1 . All others received a score of 0 . Figure 5 shows the scores for students at each grade level for the three groups.

As shown, the SG students of all levels outperformed their counterparts in the CG and PPG, and this better performance was fairly constant (as suggested by the close parallelism of the SG regression line with the CG and PPG regression lines). In other words, the conceptual scaffolding used in the study benefited students at all grade levels. In contrast, the performance of CG students was rather similar to that of the PPG students at all levels (as implied by the nearly overlapping regression lines for $\mathrm{CG}$ and PPG).

\section{STUDY 3: INVESTIGATING SUSTAINABLE EFFECTS OF SCAFFOLDING IN A LARGE-SCALE WRITTEN TEST}

\section{A. Procedure}

In the first two studies, we investigated the immediate effects of guided conceptual scaffolding on students solving synthesis problems. The third study was designed to investigate whether conceptual scaffolding would yield sustainable effects after it was removed, and whether repeated usage of scaffolded synthesis problems would improve student performance on topics not covered in the scaffolding. The latter would suggest improved ability for performing a cross-topic transfer that may better prepare students for future learning. This study was conducted in the 2009 fall quarter.
This study involved three parallel classes of a calculusbased introductory mechanics course. These classes shared the same course syllabus, materials, demonstrations, labs, recitations, quizzes, homework, midterm exams, and final exam. The instructors of all classes met weekly for more than an hour to compare and review lecture notes. They checked after each lecture to see where the other instructors were. The goal was to cover the same material at the same pace. Lectures of all instructors typically ended in the same place within $10 \mathrm{~min}$ or so. In short, the content and delivery were closely monitored to be as nearly as possible similar to what a single instructor might have done. We blindly assigned these classes into three treatment groups: SG, PPG, and control group (CTRL) containing 92, 82, and 90 students, respectively. All classes were given repeated training in the last 2 weeks (reduced to 10 days due to the overlapping of the second week with the Thanksgiving holiday) of a 10-week quarter. During this time, the SG students always received conceptual scaffolding prior to solving a synthesis problem; the PPG students always directly solved the same synthesis problems without conceptual scaffolding; and the students in the CTRL group were consistently required to solve a pair of textbooklike single-concept problems with the same topic coverage as each synthesis problem solved by the SG and PPG students. It is worth noting that the SG and PPG students received synthesis problems only for the 2-week training period. For the remaining 8 weeks of the quarter, students were mostly given traditional EOCs. Further, even during the 2-week training period students were given approximately twice as many EOCs as synthesis problems. Synthesis problems were used to augment rather than entirely replace conventional EOC problems.

In total, we used 10 sets of problem materials for each class in various learning environments, including 5 sets in lectures, 3 sets in recitations, 1 set in lab, and 1 set in homework. Take the SG class for instance: the instructor who had been trained to properly implement the materials used one set in each of the 5 lectures during the 2-week period. He followed similar procedures as outlined in study 1 and study 2, asking students to first answer the conceptual scaffolding questions and then solve a synthesis problem. A reminder to look for underlying connections was also given. Students were given time to first work on their own before the instructor modeled how to focus on deep structure rather than surface features to solve the synthesis problem. This activity took approximately $10 \mathrm{~min}$ in each lecture, accounting for $20 \%$ of the total lecture time. Anecdotal reports from the instructor showed a nearly $90 \%$ class attendance rate during the 2 weeks. Similar approaches were used for labs and recitations except that more time $(15 \mathrm{~min})$ was allocated to this activity. Also, students received feedback on how to solve the homework problems from their teaching assistants during a recitation class. Collectively, these problem 
materials covered a range of mechanics topics, including force, motion, energy, rotational dynamics, and angular momentum, with an emphasis on the last two topics due to the requirement of the course syllabus. Nevertheless, the linear momentum topic was not covered during the entire training. In all cases except for homework, time on task was controlled to be the same for the three classes.

Four days after the 2-week training, all students took the common final examination in which the synthesis problem shown in Fig. 6 was included. This problem is similar to what was used in study 2 and combines two topics broadly separated in the teaching timeline: conservation of angular momentum (a topic frequently covered in the training) and conservation of linear momentum (a topic not covered in the training). All students, regardless of which treatment class they were from, directly solved this problem without receiving any scaffolding or hints. Subsequently, student solutions were collected, blindcoded (to reduce within-grader error), and analyzed by

Tom shoots a bullet of mass $m=0.02 \mathrm{~kg}$ and initial velocity $v=300 \mathrm{~m} / \mathrm{s}$ at a wood block attached to the outer rim of a disk, which in turn rests on a horizontal frictionless air table. The bullet rips through the block, emerging in the same direction, but at a lower speed of 100 $\mathrm{m} / \mathrm{s}$. Tom sees that the mass of the wood block can be ignored, so that the disk has a moment of inertia, with $M=$ $1 \mathrm{~kg}$ and $R=25 \mathrm{~cm}$. (a) In what direction does the disk move after the collision? (b) What is the total kinetic energy of the disk after the collision?

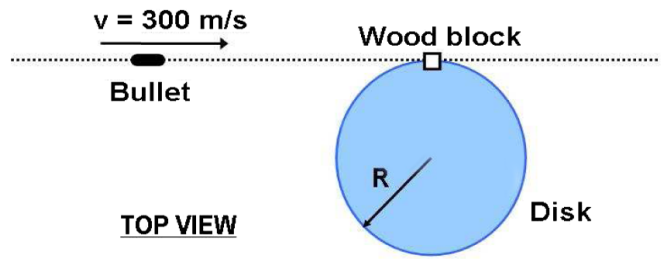

FIG. 6 (color online). A synthesis problem on the final examination. A formula for the moment of inertia of the disk $(I=$ $1 / 2 m R^{2}$ ) was given to students on a separate formula sheet. All students directly solved this problem without receiving scaffolding or other hints.

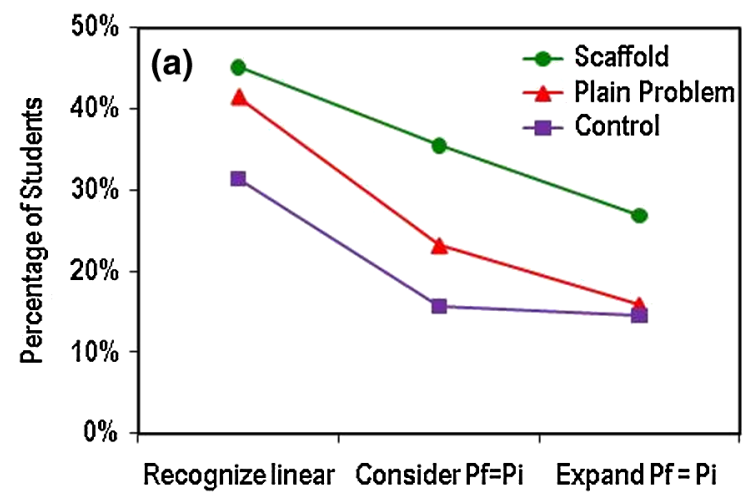

two researchers independently (to reduce between-grader error) by using the following grading schemes.

(1) Recognition.-Did the student correctly identify the motion of the disk after collision (linear and angular)? Any words, diagrams, and/or symbols that correctly expressed the motion of the disk would be considered as meeting this criterion.

(2) Consideration.-Did the student provide any written evidence showing that he or she considered using the correct fundamental principles (the linear and angular momentum conservation laws)? The same criterion as in study 2 was used.

(3) Expansion.-Did the student make any meaningful expansion of the correct fundamental principles? The same criterion as in study 2 was used.

Analysis results were then compared between the two researchers. It was found that the interrater reliability was $96 \%$, and the remaining divergence was resolved after discussion.

\section{B. Results}

Since this problem involves two topics, one covered and the other not covered in the training, we illustrate in Fig. 7 students' performance on each topic individually. As shown, for the topic of linear momentum [Fig. 7(a)] the SG students performed best in all three aspects: identification, consideration, and expansion. Using the control group as a baseline, a $\chi^{2}$ test indicates that the SG students demonstrated significantly better performance in both considering and expanding the linear momentum conservation law [consideration: $\chi^{2}(1)=9.26, p=0.002$; expansion: $\left.\chi^{2}(1)=4.15, p=0.04\right]$, and a marginally significant better performance in identifying the disk's linear motion $\left[\chi^{2}(1)=3.61, p=0.06\right]$. On the other hand, although a comparable portion of the PPG students could correctly identify the linear motion of the disk, many failed to consider invoking the pertinent conservation law, and the success rate for the PPG students to reach a meaningful expansion of this conservation law was nearly identical to that of the control group. Consequently, there was no

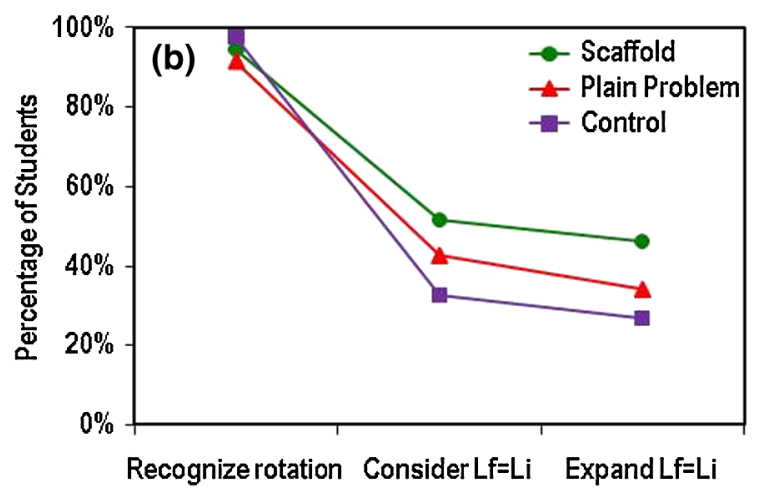

FIG. 7 (color online). Student performance on the final exam synthesis problem. (a) Performance on the linear momentum topic and (b) performance on the angular momentum topic. 
significant difference between the PPG and the control group [identification: $\chi^{2}(1)=1.85, p=0.17$; consideration: $\chi^{2}(1)=1.52, p=0.22$; expansion: $\chi^{2}(1)=0.05$, $p=0.82]$.

As for the angular momentum topic, Fig. 7(b) shows that all classes performed well in recognizing the disk's rotational motion, a phenomenon likely due to the recency effect[38], as rotational dynamics was the focus of the previous week's teaching and training. But student subsequent performance quickly diverged. The SG students again displayed the highest success rate in both considering and expanding the angular momentum conservation law. Compared to the baseline control group, the SG students' better performance was statistically significant [consideration: $\quad \chi^{2}(1)=6.75, \quad p=0.009$; expansion: $\left.\chi^{2}(1)=7.26, p=0.007\right]$. Conversely, the PPG students only achieved an intermediate success rate, comparable to that of the control group [consideration: $\chi^{2}(1)=1.86$, $p=0.17$; expansion: $\left.\chi^{2}(1)=1.04, p=0.31\right]$.

The above results indicate that in the absence of scaffolding the SG students who were trained with scaffolded synthesis problems performed highest on both topics that were covered and not covered in the training. Nevertheless, a lingering concern was that the SG students in general might have a better course background and therefore outperformed the other two classes on the synthesis problem, a possible bias in the study. To address this concern, we collected and compared students' mechanics course examination totals (two midterm and one final examination scores) in Fig. 8. As seen, students in all classes demonstrated similar course background with no evidence contesting that our initial assignment was biased in favor of the SG class over the others.

We further investigated how students at different course levels performed on the synthesis problem. We divided students into four quartiles according to their course exam totals. Also, to quantify their performance on the synthesis problem, a measure similar to that in study 2 was employed: a score of " 3 " was granted for achieving the "expansion" of both pertinent conservation laws, " 2 " for only reaching the "consideration" stage, "1" for

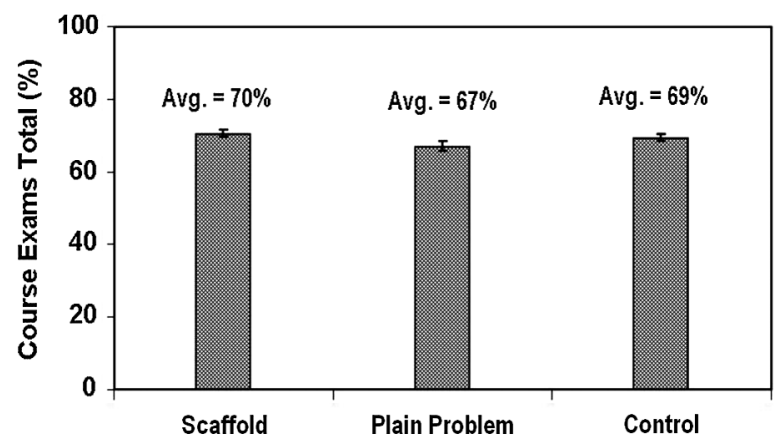

FIG. 8. Student exam totals for a calculus-based introductory mechanics course.

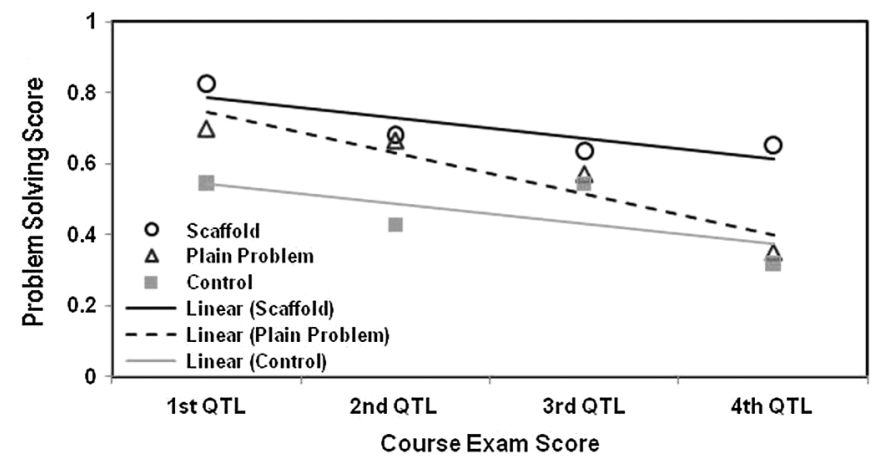

FIG. 9. Problem-solving performance for students at different course grade levels.

"recognition," and "0" for no sensible solution at all. Figure 9 shows the results for students in each quartile of the three classes. As seen, the SG students at all levels outperformed their counterparts in the control group, indicating that the repeated training with scaffolded synthesis problems benefited students at all levels. For the PPG students, however, only the top two quartiles outperformed their counterparts in the control group, and the bottom two quartiles showed no sign of advantage. This suggests that the training using synthesis problems without conceptual scaffolding selectively benefited only the higher-achieving students but not the lower-achieving students.

\section{CONCLUSIONS AND DISCUSSION}

We designed and implemented synthesis problems containing concepts that are broadly separated in the teaching timeline. Each problem was encapsulated into a sequence with two preceding conceptual questions which were related to it in terms of the same deep structure (concepts and principles). Prior to solving the problem, students were reminded to seek such relations without being told what the relations were. We implemented these questions, together with the reminder, as guided scaffolding to induce students' attention to the underlying concepts and prepare them for solving the subsequent synthesis problem.

A small-scale interview study and two large-scale written tests were conducted to explore the role of conceptual scaffolding. Results from the first two studies show that without assistance students had difficulty dealing with synthesis problems, and that using guided scaffolding can prompt students to rely on conceptual knowledge in solving physics problems. Students who answered the preceding questions were more likely to recognize deep structure and thus successfully solve the synthesis problem than those who did not receive the scaffolding. Our studies also indicate that guided conceptual scaffolding is beyond direct cueing. Students who received direct cueing could recall concepts and principles relevant to the problem at 
hand, but were less able to make a further sensible expansion than those who received conceptual scaffolding, a finding independent of students' achievement levels measured by course grades. Here, we consider the cueing on "momentum and angular momentum" as direct because it guided students to narrow their search space from a repertoire of learned knowledge down to a small number of relevant definitions or laws. On the other hand, the conceptual scaffolding used in the studies was considered as less direct, as students were not explicitly informed of any relevant concepts when answering the scaffolding questions or solving the synthesis problem.

In light of the above results, it is worth noting that a direct cue without context is less effective in helping students solve synthesis problems than the contextualized scaffolding used in our studies. One possible explanation for such a result is that the scaffolding provided a level of abstraction more suitable than direct cueing, hence facilitating student application of relevant fundamental principles. It is also possible that because the direct cueing used in the study was decontextualized it failed to provide students an opportunity to make generalization of what was cued. According to Ross and co-workers [27,39,40], being able to generalize from given cues can help solvers use deep structural features to tackle task problems. Moreover, since students receiving scaffolding could choose to work collaboratively to answer conceptual questions before solving a synthesis problem, it is also surmised that students might have benefited from peer coaching in invoking and applying the relevant principles.

Our third study exhibits the sustained effects of conceptual scaffolding on students solving synthesis problems. Students who received two weeks of training with scaffolded synthesis problems demonstrated the highest success rate on a synthesis problem in the final examination, a situation where scaffolding was completely removed. Moreover, these students performed best both on concepts that were covered (conservation of angular momentum) and not covered (conservation of linear momentum) in the training process, indicating that the trained students were better able to make a cross-topic transfer in terms of recognizing and applying deep structure to solve synthesis problems. Our correlation analysis also showed that training with scaffolded synthesis problems benefited students at all levels, whereas the training with omitted scaffolding helped only higher-achieving students. What also is interesting is that the poor performance of the control group again suggests that solving textbooklike problems does not help students to combine their learned knowledge as required for solving synthesis problems.

These results provide useful implications for effective course instruction and future research. If conceptual scaffolding can be frequently used in class to guide students' attention to deep structure, it is highly probable that they will habitually begin by searching for underlying concepts in solving physics problems. However, it is crucial that students realize and experience the unproductiveness of blind "plug-and-chug." To achieve this end, some fraction of problems must be designed in a way that students cannot solve by haphazardly grabbing locally introduced formulas. Since our ultimate goal is to train students to be independent proficient problem solvers, it is essential that we continue to seek an optimal way of removing scaffolding to further benefit students. In our studies, a sudden removal of the scaffolding after two weeks of training yielded meaningful positive results. It is conceivable that a gradual removal of the scaffolding can result in equally profitable outcomes. Also, it can be beneficial to further investigate the effects of problem surface features on students' performance by fine-tuning the similarities between scaffolding questions and synthesis problems [27,39,40]. This work may help identify effective means of manipulating certain features to maximally facilitate students' access and instantiation of fundamental principles in solving synthesis problems. Moreover, the grading rubrics used in the present study, albeit useful, were rudimentary. In future studies, more detailed grading rubrics [41] may be developed to examine student problem solving at a finer grain level.

\section{ACKNOWLEDGMENTS}

The authors wish to thank Ken Heller, Noah Podolesky, and the three anonymous reviewers for their insightful comments. The authors also appreciate the support of the School of Teaching and Learning and the Department of Physics at the Ohio State University (OSU). This study is partially supported by The Ohio State University EHE SEED grant and National Science Foundation Grant No. 0618128.
[1] A. Van Heuvelen, Learning to think like a physicist: A review of research based instructional strategies, Am. J. Phys. 59, 891 (1991).

[2] D. Maloney, in Handbook of Research on Science Teaching and Learning Project, edited by D. Gabel (MacMillan, London, 1994).
[3] L. McDermott, Oersted Medal Lecture 2001: Physics education research-the key to student learning, Am. J. Phys. 69, 1127 (2001).

[4] P. Heller and K. Heller, Cooperative Group Problem Solving in Physics (University of Minnesota, Minnesota, 2001). 
[5] N. Rebello, L. Cui, A. Bennett, D. Zollman, and D. Ozimek, in Learning to Solve Complex Scientific Problems, edited by D. Jonassen (Lawrence Earlbaum, Mahwah, NJ, 2007).

[6] P. Kohl and N. Finkelstein, Patterns of multiple representation use by experts and novices during physics problem solving, Phys. Rev. ST Phys. Educ. Res. 4, 010111 (2008).

[7] J. Larkin and F. Reif, Understanding and teaching problem-solving in physics, Eur. J. Sci. Educ. 1, 191 (1979).

[8] J. Larkin, Processing information for effective problem solving, Eng. Educ. 70, 285 (1979).

[9] J. Larkin, Skilled problem solving in physics: A hierarchical planning model, J. Struct. Learn. 6, 271 (1980).

[10] F. Reif, Teaching problem solving-A scientific approach, Phys. Teach. 19, 310 (1981).

[11] J. Larkin, J. McDermott, D. Simon, and H. Simon, Expert and novice performance in solving physics problems, Science 208, 1335 (1980).

[12] M. Chi, P. Feltovich, and R. Glaser, Categorization and representation of physics problems by experts and novices, Cogn. Sci. 5, 121 (1981).

[13] P. Hardiman, R. Dufresne, and J. Mestre, The relation between problem categorization and problem solving among experts and novices, Mem. Cogn. 17, 627 (1989).

[14] T. de Jong, and M. Ferguson-Hessler, Cognitive structures of good and poor novice problem solvers in physics, J. Educ. Psychol. 78, 279 (1986).

[15] T. de Jong and M. Ferguson-Hessler, Knowledge of problem situations in physics: A comparison of good and poor novice problem solvers, Learn. Instr. 1, 289 (1991).

[16] E. R. Savelsbergh, T. de Jong, and M. Ferguson-Hessler, Situational knowledge in physics: The case of electrodynamics, J. Res. Sci. Teach. 39, 928 (2002).

[17] W. Gerace and I. Beatty, Teaching vs. Learning: Changing perspectives on problem solving in physics instruction, in Proceedings of the 9th Common Conference of the Cyprus Physics Association and Greek Physics Association, Nicosia, Cyprus, 2005 (unpublished).

[18] L. McDermott, Millikan Lecture 1990: What we teach and what is learned-Closing the gap, Am. J. Phys. 59, 301 (1991).

[19] E. Kim and S. Pak, Students do not overcome conceptual difficulties after solving 1000 traditional problems, Am. J. Phys. 70, 759 (2002).

[20] F. Reif and L. Scott, Teaching scientific thinking skills: Students and computers coaching each other, Am. J. Phys. 67, 819 (1999).

[21] R. Dufresne, W. Gerace, P. Hardiman, and J. Mestre, Constraining novices to perform expertlike problem analyses: Effects on schema acquisition, J. Learn. Sci. 2, 307 (1992).

[22] J. Mestre, R. Dufresne, W. Gerace, and P. Hardiman, Promoting skilled problem-solving behavior among beginning physics students, J. Res. Sci. Teach. 30, 303 (1993).

[23] W. Leonard, R. Dufresne, and J. Mestre, Using qualitative problem-solving strategies to highlight the role of conceptual knowledge in solving problems, Am. J. Phys. 64, 1495 (1996).
[24] S. Kanim, An investigation of student difficulties in qualitative and quantitative problem solving: Examples from electric circuits and electrostatics, Ph.D. thesis, University of Washington, 1999.

[25] C. Singh, Assessing student expertise in introductory physics with isomorphic problems. II. Effects of some potential factors on problem solving and transfer, Phys. Rev. ST Phys. Educ. Res. 4, 010105 (2008).

[26] M. Gick and K. Holyoak, Analogical problem solving, Cogn. Psychol. 12, 306 (1980).

[27] B. Ross and P. Kennedy, Generalizing from the use of earlier examples in problem solving, J. Exp. Psychol. Learn. Mem. Cogn. 16, 42 (1990).

[28] J. Larkin, J. McDermott, D. Simon, and H. Simon, Model of competence in solving physics problems, Cogn. Sci. $\mathbf{4}$, 317 (1980).

[29] Although a "forward" strategy is not always a preferred approach to problem solving, students' working forward in this study resulted from their working from the relevant fundamental concepts. Readers can find descriptions of metalevel backward and plan-stacking problem-solving strategies in A. G. Preset and R. O. Lindsay, New light on novice-expert differences in physics problem solving, Br. J. Psychol. 83, 389 (1992).

[30] L. Walsh, R. Howard, and B. Bowe, Phenomenographic study of students' problem solving approaches in physics, Phys. Rev. ST Phys. Educ. Res. 3, 020108 (2007).

[31] One could choose to set up a differential equation for the motion of the damped oscillation. But given the nature of the frictional force (constant magnitude and alternating direction), it would not be possible to solve the differential equation by hand. The authors have tried VPYTHON to numerically solve the problem. Interested readers can also use VPYTHON or other computational software to verify the oscillator's distance $(3.8 \mathrm{~m})$ and position $(+0.6 \mathrm{~m})$ after being released from the initial maximum stretch of the spring $(1 \mathrm{~m})$.

[32] J. Tuminaro and E. Redish, Elements of a cognitive model of physics problem solving: Epistemic games, Phys. Rev. ST Phys. Educ. Res. 3, 020101 (2007).

[33] A. Newell and H. A. Simon, Human Problem Solving (Prentice-Hall, Englewood Cliffs, NJ, 1972).

[34] K. Heller (private communication).

[35] This problem is further complicated by the fact that after collision the center of mass of the disk-bob system is not at the disk center. In the third study we simplified the situation by letting a bullet exit the catcher at a lower speed.

[36] This is a typical sequence similar to what appears in some widely adopted texts. See, for example, R. D. Knight, Physics for Scientists and Engineers: A Strategic Approach (Pearson Addison-Wesley, San Francisco, 2004).

[37] We used the following grade point conversion method: $\mathrm{A}=4.0, \mathrm{~A}-=3.7, \mathrm{~B}+=3.3, \mathrm{~B}=3.0, \mathrm{~B}-=2.7$, $\mathrm{C}+=2.3, \mathrm{C}=2.0, \mathrm{C}-=1.7, \mathrm{D}+=1.3, \mathrm{D}=1.0$, $\mathrm{D}-=0.7$ and $\mathrm{E}=0$, equivalent to "fail."

[38] A. Baddeley and G. Hitch, The recency effect: Implicit learning with explicit retrieval, Mem. Cogn. 21, 146 (1993). 
[39] B. Ross, This is like that: The use of earlier problems and the separation of similarity effects, J. Exp. Psychol. Learn. Mem. Cogn. 13, 629 (1987).

[40] B. Ross, Distinguishing types of superficial similarities: Different effects on the access and use of earlier problems,
J. Exp. Psychol. Learn. Mem. Cogn. 15, 456 (1989).

[41] J. Docktor, Development and validation of a physics problem-solving assessment rubric, Ph.D. thesis, University of Minnesota, 2009 (unpublished). 